\title{
Blockbuster dreams for Pfizer's CDK inhibitor
}

Dramatic clinical data seen recently with Pfizer's leading small-molecule CDK4/6 inhibitor palbociclib (PD-0332991) has rekindled interest in the concept of blocking cell cycle progression to stop cancer cell growth. Cyclin-dependent kinase (CDK) inhibitors have for many years failed to reach the market, despite a strong mechanistic rationale to back their use. The New York-based big pharma plans to start a phase 3 trial with palbociclib in advanced, post-menopausal, estrogen receptor (ER)-positive breast cancer in the first half of this year. The decision follows phase 2 interim results presented at the CTRCAACR San Antonio Breast Cancer Symposium last December, showing that the oral drug prolonged median progression-free survival (PFS) by over 18 months in the randomized two-part trial which included 165 patients with advanced or metastatic ER-positive, HER2-negative breast cancer. When palbociclib was added to the standard anti-hormonal agent Femara (letrozole), from Basel-based Novartis, the median PFS improved from 7.5 months with Femara alone to 26.1 months with the combination (according to Pfizer, these data are not finalized and continue to be analyzed).

Some analysts believe that the drug has multibillion dollar sales potential, with Mark Schoenebaum at ISI Group, New York, stating that it has blockbuster potential in ER-positive breast cancer alone, given the strength of the data and large patient population. However, analyst Tony Butler at Barclays Capital in New
York cautiously says that he is awaiting confirmation that it improves overall survival.

The palbociclib data bode especially well for the other CDK4/6 inhibitors in earlier clinical development, according to researchers. These include Lilly's LY2835219, which will soon begin phase 2 development in mantle cell lymphoma (MCL), and Novartis' LEE011. The drugs inhibit phosphorylation of the retinoblastoma protein in the early G1 phase of the cell cycle, blocking progression to $\mathrm{S}$ phase, where DNA is replicated.

Whether other CDK inhibitors working by different mechanisms will show the same benefits as CDK4/6 inhibitors remains unproven. "The main issue is to understand which CDKs should be targeted in specific cancers where they have a key role in the oncogenic process," says Antonio Giordano of Temple University in Philadelphia. Merck believes, for example, that dinaciclib, in phase 3 development in chronic lymphocytic leukemia (CLL), works mainly through inhibiting transcription of antiapoptotic proteins by blocking CDK9, as does Astex Pharmaceuticals of Cambridge, UK, of its pan-CDK inhibitor AT7519, in phase 2 trials in multiple myeloma, CLL and MCL. Meanwhile, Spiro Rombotis, president and CEO of Cyclacel, in Berkeley Heights, New Jersey, points to the CDK2-dominant activity of its first-generation CDK inhibitor seliciclib and its more potent second-generation, preclinical compound CYC065. "The palbociclib data are very

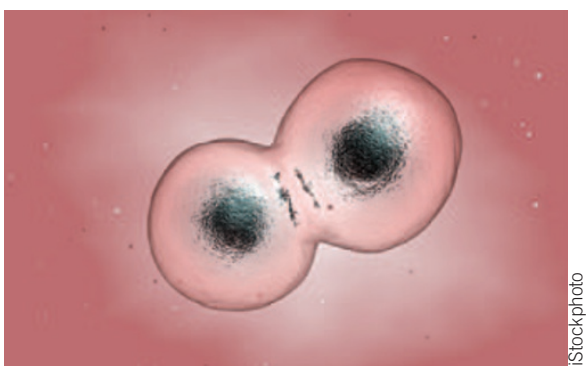

Cell cycle inhibitors back in vogue.

encouraging. However, the different CDK isoforms might be useful in different indications," he says. "One size does not fit all." At the DanaFarber Cancer Institute in Boston, Geoffrey Shapiro responds to the latest data by pointing out "it will be important to examine mechanisms of resistance to CDK4/6 drugs, and one of these may be through CDK2 activation."

Researchers generally believe that biomarkerbased selection of patients will be critical to the different CDK inhibitors, demonstrating efficacy in different tumor types. "The Pfizer trial showed that if you find the right patient population, you get the activity you expect," says Konstantin Dragnev of the Geisel School of Medicine at Dartmouth in Hanover, New Hampshire. Shapiro points to HER2-positive breast cancer and KRAS-mutant non-small cell lung cancer, for example, as other tumor subtypes to explore with CDK4/6 inhibitors, based on preclinical data and mechanistic rationale.

Malini Guha, Freehold, New Jersey

\section{Around the world in a month}

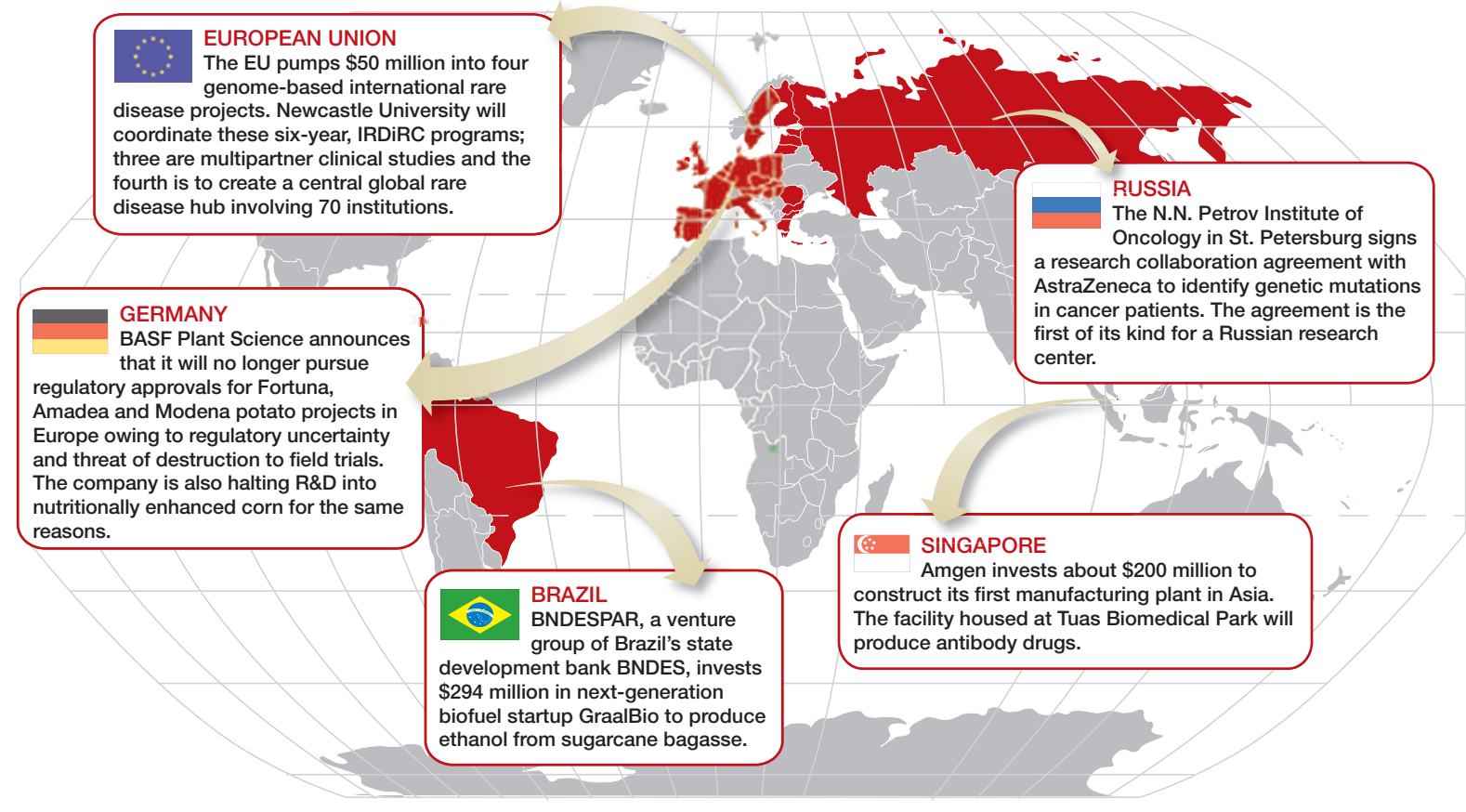

